\title{
The Effects of Aqueous Leave Extract of Moringa oleifera on the Hippocamppal Histology of Aluminium Chloride-Induced Alzheimer's Disease in Adult Wistar Rats
}

\author{
Ignatius Ikemefuna Ozor ${ }^{1}$, Zita Njideka Agwagu ${ }^{2}$, Elizabeth Finbarrs-Bello ${ }^{1}$, \\ Onyinye Mary Ozioko ${ }^{1,}$, Uche Sebastine Ozioko ${ }^{1}$, Naza Christian Iruka ${ }^{1}$ \\ ${ }^{1}$ Department of Anatomy, College of Medicine, Enugu State University of Science and Technology, Enugu, Nigeria \\ ${ }^{2}$ Department of Nursing, Enugu State University of Science and Technology Teaching Hospital, Enugu, Nigeria
}

Email address:

oziokoonyinye@gmail.com (O. M. Ozioko)

${ }^{*}$ Corresponding author

\section{To cite this article:}

Ignatius Ikemefuna Ozor, Zita Njideka Agwagu, Elizabeth Finbarrs-Bello, Onyinye Mary Ozioko, Uche Sebastine Ozioko, Naza Christian Iruka. The Effects of Aqueous Leave Extract of Moringa oleifera on the Hippocamppal Histology of Aluminium Chloride-Induced Alzheimer's Disease in Adult Wistar Rats. International Journal of Neurologic Physical Therapy. Vol. 6, No. 2, 2020, pp. 23-28. doi: 10.11648 j.ijnpt.20200602.12

Received: July 30, 2020; Accepted: August 17, 2020; Published: September 10, 2020

\begin{abstract}
Introduction: Consumption of antioxidant-rich foods and polyphenol treatment can enhance cognitive performance in elderly subjects. Hence, this study was aimed at investigating the effects of aqueous extract of Moringa oleifera leaves on the hippocampus of aluminium chloride-induced alzheimer's disease in adult wistar rats. Method: Twenty (20) healthy adult male wistar rats (Rattus norvegicus), weighing 180-240g were divided into five (5) groups of four (4) animals each. Group one served as normal control group and was given free access to normal saline for 21 days, group 2 was given 400 $\mathrm{mg} / \mathrm{kg}$ of Moringa extract only for 21 days, group 3 was given $200 \mathrm{mg} / \mathrm{kg}$ of $\mathrm{AlCl}_{3}$ for 21 days. Group 4 which served as treatment group was given co-treatment of Moringa extract and $\mathrm{AlCl}_{3}$ for 21 days while groups 5 which served as standard drug group received co-treatment of Simvastatin and $\mathrm{AlCl} 3$ for 21 days. Parameters studied include; hippocampal CA3 histopathology, and CA3 histochemical changes after H\&E and Congo red stains. Results: This study revealed that aqueous leaves extract of Moringa oleifera and standard drug-Simvastatin- at both Histopathological and histochemical parameter was effective. Moringa aqueous extracts ameliorated the reported histochemical and histopathological effects of $\mathrm{AlCl}_{3}$ in treated rats. Conclusion: This study reveals that the tested extracts provided efficiently a neuroprotective effect against Alzheimer's disease. The Moringa extract group and Simvastatin group showed effectiveness in treatment of neurodegenerative disease. This shows that Moringa extract is an alternative remedy to Simvastatin in the treatment of neurodegenerative disease.
\end{abstract}

Keywords: Alzheimer's Disease, Hippocampus, Moringa oleifera

\section{Introduction}

Dementia is a clinical syndrome characterized with memory, attention, language, and problem solving impairments affecting the daily activity living of patients. Alzheimer's disease (AD) is the most common neurodegenerative disease affecting more than 40 million people worldwide [1]. Alzheimer's disease is the most common cause of progressive dementia in the elderly population. It is a chronic neurodegenerative disorder that leads to progressive disturbances of cognitive functions including memory, judgement, decision-making, orientation to physical surroundings and language [2]. Alzheimer's Disease (AD) has been postulated to result from oxidative stress elevation in the brain [3], extracellular formation of amyloid plaques and intracellular deposits of neurofibrilary tangles in the hippocampus, cerebral cortex [4] Acetylcholinesterase (AChE) inhibitors are the most common class of drugs for $\mathrm{AD}$, such as galantamine, rivastigmine, and donepezil, which temporarily enhance the availability of acetylcholine at cholinergic synapses $[5]$. 
The hippocampus is a small, curved formation in the brain that plays an important role in the limbic system [6]. It is found in the temporal lobe below the cerebral cortex $[7,8]$. In adult humans, the volume of the hippocampus on each side of the brain is about $3-3.5 \mathrm{~cm}^{3}$ as compared to $320-420$ $\mathrm{cm}^{3}$ for the volume of the neocortex [6]. The hippocampal formation is a key site of pathology in Alzheimer's disease [9] and its clinical consequence has been extensively studied [10, $11]$.

Aluminum (Al) is the third most common, and one of the most widely distributed metallic elements in the earth's crust $[12,13]$. Although most $\mathrm{Al}$ exists as insoluble aluminosilicates and $\mathrm{Al}$ oxides, it is a known neurotoxin which presents an opportunity for human exposure and provides ubiquitous contamination [14]. Many reports implicate Al with Alzheimer's Disease (AD), Amyotrophic Lateral Sclero [3] sis (ALS), Parkinsonism Dementia Complex, etc. [15-17]. Al is capable of producing much damage to the nervous system, including the impairment of learning and memory [18].

Statins are a class of medications that reduce cholesterol by inhibiting 3-hydroxy-3-methylglutaryl-coenzyme A reductase [19]. Statins is commonly known as 3-hydroxy-3methylglutaryl coenzyme A reductase inhibitors, and is the first-line drug therapy for the treatment of hyperlipidemia and the first choice for the prevention of coronary heart disease [20]. Its clinical application include reducing blood lipids however prevention and treatment of dementia is gradually drawing people's attention [21]. Statins not only lower serum cholesterol levels, but also inhibit pivotal enzymatic reactions (e.g. the isoprenylation of a subset of GTPases) [22] that lead to amyloid deposition and plaque formation; both are considered cornerstone pathways underpinning the development of Alzheimer's disease (AD) [23].

Moringa oleifera is a miracle tree species [24] with so many nutrients, consumption of $M$. oleifera leaf reinforces neural response, stimulates immune functions, and improves health because of the large amounts of microelements and polyphenol antioxidants [25]. Accumulative lines of evidence have demonstrated that consumption of antioxidant-rich foods and polyphenol treatment can enhance Anxiolytic and curative effects in the brain [26-32] and has also been reported to combat oxidative stress in rat model of Alzheimer's disease induced by colchicines such as vitamin $\mathrm{C}$ and vitamin E [33]. However, the scientific evidence concerning the effect of $M$. oleifera leaves extract on cognitive dysfunction induced by hypocholinergic function remains limited.

\section{Materials and Methods}

\subsection{Sample Preparation and Extraction Procedure}

\subsubsection{Plant Materials}

Fresh leaves of the plant, Moringa oleifera was harvested from plants grown in Enugu State, Nigeria. The leaves were identified by a crop scientist of the Department of Agronomy and Ecological Management, Faculty of Agriculture and Natural resources Management, Enugu State University of Science and Technology, Agbani, Enugu, Nigeria on January 2020 .

\subsubsection{Preparation of Moringa oleifera Extract}

The sample was dried in air for 7 days and after complete drying was crushed in the form of fine powder with a super Sony Japan electric blender. $200 \mathrm{~g}$ of dried plant extract was immersed in $2000 \mathrm{mls}$ of water and was evaporated with a Soxhlet extractor to yield $21.2 \mathrm{~g}$ of gel extract, which was refrigerated until ready for use [34]. The experimental chemical Aluminium Chloride was procured from an approved pharmaceutical/chemical store in Enugu metropolis.

\subsubsection{Animal Management and Grouping}

The study was approved by the Ethical Committee of Enugu State University of Science and Technology, College of Medicine and carried out in accordance with the principles of laboratory animal care and standard experimental procedures.

Twenty four Wistar rats at the age of approximately three months each weighing 180-240g, were used for the study. The animals were kept in a plastic cage with iron nettings placed in a well ventilated standard housing conditions, twelve hours light and twelve hours darkness. The animals were acclimatized for two weeks before the commencement of the experiment. During this period, the animals were observed to ensure that they were disease free. They were fed with rat chows and given water ad libitum, and an ambient temperature range of 25-27 maintained at 50\% humidity. The animals were weighed with an electronic weighing balance prior to the commencement and termination of the experiment.

\subsection{Experimental Design}

After an acclimatization, the rats were divided into five groups each consisting of four animals in accordance to their body weight and the experiment was run for twenty one days. Rats were administered their respective doses of Moringa oleifera, $\mathrm{AlCl}_{3}$, and Simvastatin each for 21days. The groups (A-F): A (positive control giving normal saline only for 21 days), B (Moringa extract for 21 days), C (negative control giving Aluminium Chloride for 21 days), D (Aluminium Chloride and Moringa oleifera extract co-treatment for 21 days), and standard group E (Aluminium Chloride and Simvastatin for 21 days).

Each group consisted of four rats. Alzheimer's disease was induced (in groups $\mathrm{C}, \mathrm{D}$ and $\mathrm{E}$ 'test' rats) by oral gavage administration of $\mathrm{Alcl}_{3}(200 \mathrm{mg} / \mathrm{kg}$ body weight), freshly dissolved in $0.9 \%$ saline. The positive control rats were injected with only $0.9 \%$ saline orally.

The brain analysis was carried out to check effect of the aqueous extracts of Moringa oleifera leaves on the Hippocampus.

The histological examination was also carried out to 
determine the extent of degeneration, regeneration, ameliorative and curative effect of the Moringa oleifera extract, the deposition of beta amyloid, and its curative and attenuation in comparison to the positive control.

Table 1. Table showing grouping of experimental animals.

\begin{tabular}{|c|c|c|c|c|}
\hline Groups & No of rats per cage & Inducing agent/ extract & Dosage & Duration \\
\hline Group A (positive control group) & 4 & Normal saline & Control & 21 days \\
\hline Group B (negative control) & 4 & MOE extract & $400 \mathrm{mg} / \mathrm{kg}$ & 21 days \\
\hline Group C & 4 & $\mathrm{AlCl}_{3}$ & $200 \mathrm{mg} / \mathrm{kg}$ body weight & 21 days \\
\hline Group D & 4 & $\mathrm{AlCl}_{3}+\mathrm{MOE}$ extract 3 simultaneously & $\mathrm{AlCl}_{3}=200 \mathrm{mg} / \mathrm{kg}, \mathrm{MOE}=400 \mathrm{mg} / \mathrm{kg}$ & 21days \\
\hline Group E & 4 & $\mathrm{AlCl}_{3}+$ Simvastatin & $\mathrm{AlCl}_{3}=200 \mathrm{mg} / \mathrm{kg}$, Simvastatin $=40 \mathrm{mg} / \mathrm{kg}$ & 21 days \\
\hline
\end{tabular}

\subsection{Animal Sacrifice, Tissue Collection and Processing}

Before sacrificing the wistar rats, the final weight of the test animals were collected. The wistar rats were anaesthetized using chloroform. The cranium of each of the animals was opened using brain Forceps, the hippocampus was then separated from the cerebrum. The tissues were then fixed in $10 \%$ formal saline and processed for histological observation using routine Haematoxylin and Eosin, and Congo red staining techniques.

\subsection{Tissue Preparation for Microscopy}

The tissue were subsequently trimmed, dehydrated in ascending grades of alcohol $(70 \%, 80 \%, 90 \%$ and absolute alcohol), cleared in three (3) changes of xylene and embedded in molten paraffin wax. Sections of 5 um thickness with a rotary microtome were made, floated in water bath (45oc) and incubated at 60 oc to dry. The 5um thick sections were subsequently stained in using hematoxylin and Eosin stains. The prepared slides was examined with a Motic ${ }^{\mathrm{TM}}$ compound light microscope using $\mathrm{x} 4, \mathrm{x} 10$ and $\mathrm{x} 40$ objective lenses. The photomicrographs was taken using a Motic ${ }^{\text {TM }} 2.0$ megapixels microscope camera at x100; x160 \& x400 magnifications [35].

\section{Results}

\subsection{Hippocampus (H and $E)$}

Representative photomicrographs of the hippocampus showing the panoramic view of the CA3 region (Cornu Ammonis). The CA3 is the region with large densely packed pyramidal cells (black arrow) in the concavity of the dentate gyrus. Deducible histology presentation includes; cellular delineation, cellular density, staining intensity and histomorphology cellularity. Two copies of images are made available one of which is labelled and the other is plain.

\subsection{Hippocampus (Congo Red)}

Representative photomicrographs of the hippocampus showing deposition of amyloid $\beta$ plaques (yellow arrow) in the CA3 region at a high magnification (X400). Slides presents with varying staining intensity and cellular density. Amyloid deposit is stained red and nuclei are stained blue. Two copies of images are made available one of which is labelled and the other is plain.

\section{Discussion}

Poor neuronal connectivity, neuronal loss in the overall brain matter with specific loss of cerebellar and hippocampal neurons, decreased dendritic spine density and remodeling could lead to impaired performance on a hippocampaldependent learning and memory task, context discrimination [36] and memory disturbances which are clinical signs in $\mathrm{AD}$, even in the preclinical stages [37]. In this study (Figures 2, 3 and 5) the photomicrographs showed shrunken pyramidal cells which is an evidence of neurodegenerative disease [38] post chronic administration of the Moringa Leaves extract in the wistar rat.

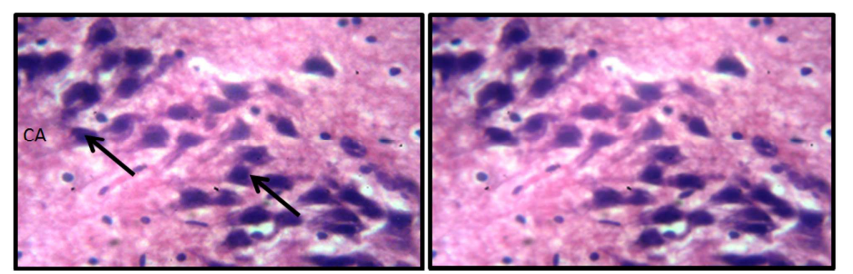

Figure 1. (Normal control): Representative photomicrograph of the CA3 region of the hippocampus of experimental animal. The Haematoxylin and Eosin photomicrograph shows normal histomorphology and staining intensity of the densely and tightly packed pyramidal cells of the Cornu Ammonis (CA3) region (H and Ex400).

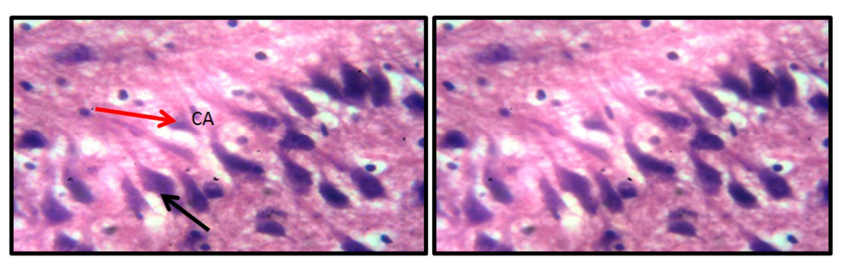

Figure 2. (Moringa group): Representative photomicrograph of the CA3 region of the hippocampus of experimental animal. The Haematoxylin and Eosin photomicrograph shows normal histomorphology and staining intensity but the pyramidal cells of the Cornu Ammonis 3 region are not densely and tightly packed. Few shrunken cells are present (red arrow) (H and E $x 400$ ).

In this study, (Figure 3) histopathological evaluation of hippocampal sections of $\mathrm{AlCl} 3$ treated group of rats confirmed neurodegenerative changes in CA3. This is consistent with previous study by [39] where $\mathrm{AlCl} 3$ induction group showed massive depletion of cellular body, acidic cytoplasm, nuclear vacuolation and more intercellular space. However, the experimental group (Moringa extract $+\mathrm{AlCl} 3$ ) (Figure 4) showed fewer shrunken cells than in Figure 3. 
This shows that the administration of Moringa extract was able to attenuate the pathological changes of pyramidal cells in the CA3 region, in the Co-treatment.

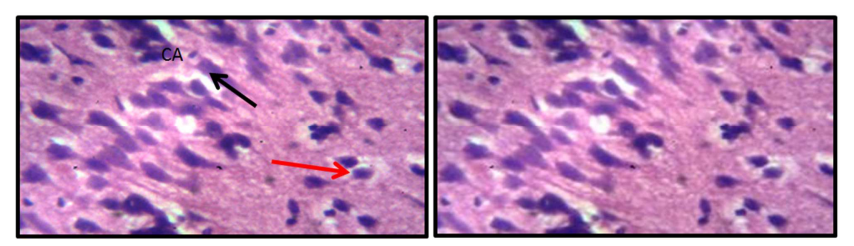

Figure 3. $\left(\mathrm{AlCl}_{3}\right.$ group): Representative photomicrograph of the $\mathrm{CA} 3$ region of the hippocampus of experimental animal. The Haematoxylin and Eosin photomicrograph shows normal histomorphology and staining intensity but the pyramidal cells of the Cornu Ammonis 3 region are not densely and tightly packed. Few shrunken cells are present (red arrow) (H and E x400)

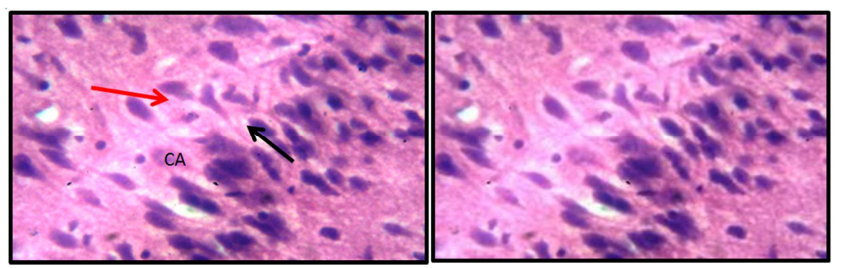

Figure 4. (Moringa and $\mathrm{AlCl}_{3}$ group): Representative photomicrograph of the CA3 region of the hippocampus of experimental animal. The Haematoxylin and Eosin photomicrograph shows normal histomorphology and staining intensity of the densely and tightly packed pyramidal cells of the Cornu Ammonis 3 region. Few shrunken cells are present (red arrow) (H and Ex400).
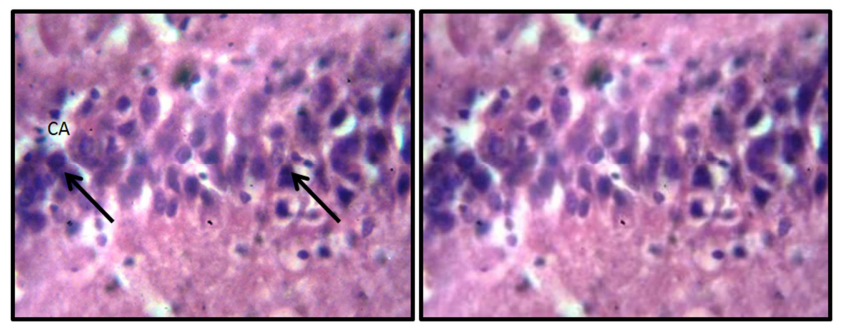

Figure 5. (Moringa extract and Simvastatin): Representative photomicrograph of the CA3 region of the hippocampus of experimental animal. The Haematoxylin and Eosin photomicrograph shows normal histomorphology and staining intensity of the densely and tightly packed shrunken pyramidal cells of the Cornu Ammonis 3 region. (H and E x400).

\subsection{Hippocampus (Congo Red)}

The histochemical changes in hippocampus CA3 region was examined with Congo red stain. Congo red stain demonstrated amyloid deposits in tissue sections. The normal saline treated groups (Figure 6) present normal histology and normal neuronal cells implying that there is no deposition of amyloid $\beta$ plaque. The photomicrography of $\mathrm{AlCl}_{3}$ treated wistar rats slide (Figure 8) showed high extracellular deposition of preformed senile plaques with hollow-spaced neuropils in pyramidal cells of hippocampus (CA3) suggesting Alzheimer's disease. This is in agreement with the previous study of [40] which identified the presence of beta amyloid plaque as hallmark pathology required for a diagnosis of Alzheimer's disease.

The Moringa extract treated group (Figure 7) in this study showed moderate deposition of amyloid $\beta$ plaques suggesting neurotoxicity in chronic consumption of overdose of Moringa. This implies that chronic consumption of Moringa at high dose has negative side effects on the body as also supported by the results from the H\&E stain of the present study.

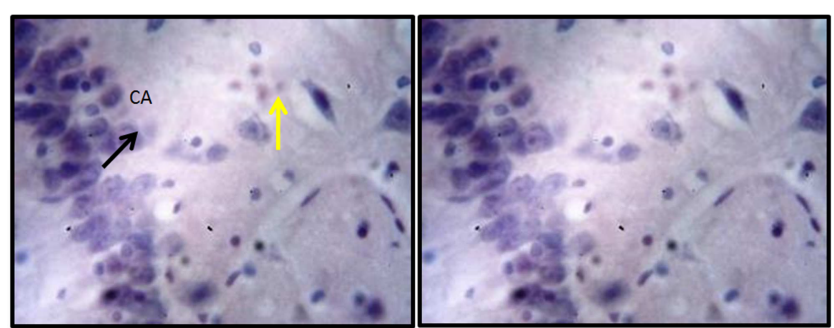

Figure 6. (Normal control): Representative photomicrograph of the CA3 region of the hippocampus of experimental animal showing pyramidal cells. This slide present pyramidal cells that are densely packed and properly delineated. Normal histology and normal neuronal cells. (Congo red X400).

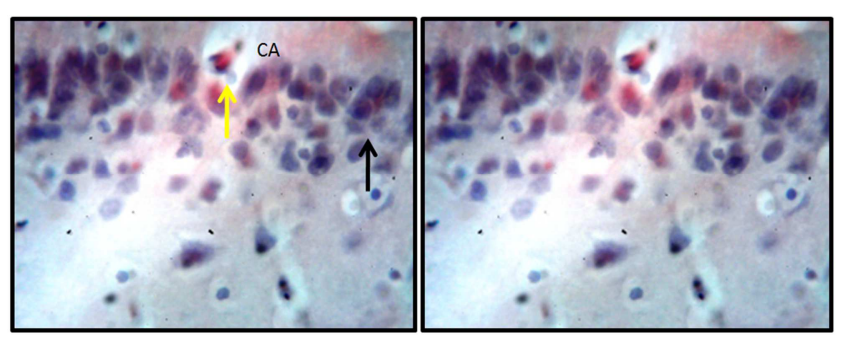

Figure 7. (Moringa group): Representative photomicrograph of the CA3 region of the hippocampus of experimental animal showing pyramidal cells. This slide present pyramidal cells that are densely packed and properly delineated. Moderate deposition of amyloid $\beta$ plaques (yellow arrow) (Congo red $X 400)$.

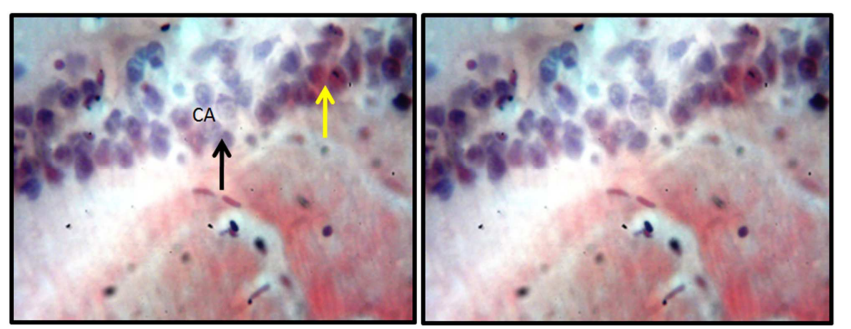

Figure 8. ( $\mathrm{AlCl}_{3}$ group): Representative photomicrograph of the $\mathrm{CA} 3$ region of the hippocampus of experimental animal showing pyramidal cells. This slide present pyramidal cells that are densely packed and properly delineated. High deposition of amyloid $\beta$ plaques (yellow arrow) (Congo red X400).

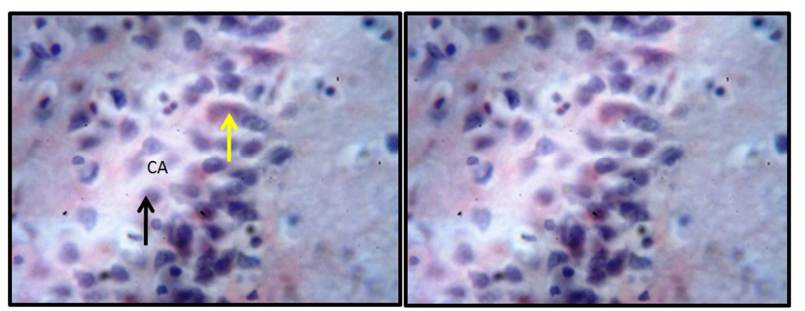

Figure 9. (Moringa and $\mathrm{AlCl}_{3}$ group): Representative photomicrograph of the CA3 region of the hippocampus of experimental animal showing pyramidal cells. This slide present pyramidal cells that are densely packed and properly delineated. Moderate deposition of amyloid $\beta$ plaques (yellow arrow). (Congo red X400). 


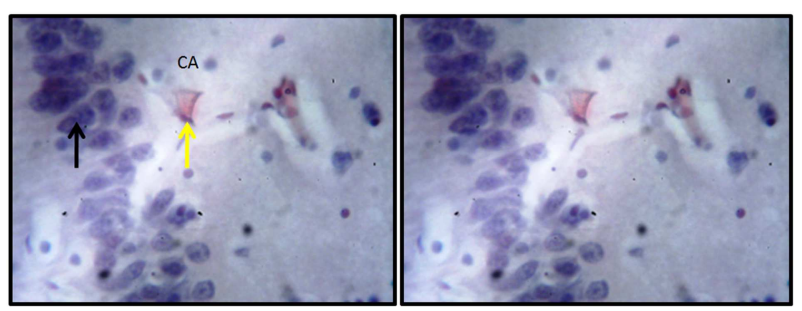

Figure 10. (Moringa extract and Simvastatin): Representative photomicrograph of the CA3 region of the hippocampus of experimental animal showing pyramidal cells. This slide present pyramidal cells that are densely packed and properly delineated. Moderate deposition of amyloid $\beta$ plaques (yellow arrow) (Congo red X400).

Moringa extract and $\mathrm{AlCl}_{3}$ co-treatment in this study (Figure 9), showed moderate deposition of amyloid $\beta$ plaques, signifying that administration of Moringa extract attenuated the pathological changes induced by the $\mathrm{AlCl}_{3}$. The co-treatment of Simvastatin and $\mathrm{AlCl} 3$ (Group E) showed moderate deposition of amyloid $\beta$ plaques implying that the Simvastatin was able to ameliorate the pathological effects of $\mathrm{AlCl}_{3}$.

\subsection{Comparison of Simvastatin with Moringa Leave Extract}

Accumulative lines of evidence have demonstrated that consumption of antioxidant-rich foods has also been reported to combat oxidative stress in rat model of Alzheimer's disease induced by colchicines such as vitamin $\mathrm{C}$ and vitamin E [33]. H\&E stain, (Figure 1 - Figure 5) result gotten from our study showed normal histomorphology and staining intensity but few shrunken pyramidal cells in both moringa extract and simvastatin. This implies that both remedies ameliorated neuronal degeneration.

From the result gotten from the Congo red stain, moderate deposition of amyloid $\beta$ plaques in the photomicrographs of both Moringa oleifera extract group and simvastatin group was observed. This implies that both Moringa and Simvastatin attenuated the accumulation of amyloid $\beta$ plaque in the nerve cell of the brain.

It can be deduced therefore that both Moringa Extract and Simvastatin ameliorated neuronal degeneration and as well attenuated the accumulation of amyloid $\beta$ plaque hence Moringa oleifera extract can be used as an alternative drug to Simvastatin in the cure of Alzheimer's disease.

\section{Conclusion}

Herbal remedies have been on the increase especially due to side effects and high cost of some conventional medicines. The extract of Moringa oleifera despite its numerous benefits in treatment of various ailments also showed convincing evidence as a remedy for neurodegenerative diseases. The leave extract of Moringa oleifera application on the Alzheimer's disease-induced Wistar rat ameliorated the neurodegeneration and showed its protection against the disease. Thus advocate for the use of Moringa leave extract in the control and management of neurodegenerative diseases such as the Alzheimer's disease.
Further study should however be carried out to determine dose and duration dependent effect administration of Moringa oleifera extract on the ultrastructural changes in hippocampal histology and neuronal synaptogenesis.

\section{References}

[1] Alzheimer's Disease International, (2018). World Alzheimer ReportsAlzheimer'sDiseaseInternational. Availableat: https://www.alz.co.uk/research/world-report (accessed October 30, 2019).

[2] Nussbaum RL, Ellis CE. 2003 Alzheimer's disease and Parkinson's disease. N Engl J Med; 348: 1356-64.

[3] Mattson, M. P. 2004. Pathways towards and away from alzheimer's disease. Nature. 430 (7000) 631-639.

[4] Da Rocha, M. D., Viegas, F. P., Campos, H. C., Nicastro, P. C., Fossaluzza, P. C., Fraga, C. A., Barreiro, E. J., \& Viegas, C., Jr (2011). The role of natural products in the discovery of new drug candidates for the treatment of neurodegenerative disorders II: Alzheimer's disease. CNS \& neurological disorders drug targets, 10 (2), 251-270.

[5] Grossberg G. T. 2003. Cholinesterase inhibitors for the treatment of Alzheimer's disease: getting on and staying on. Current therapeutic research, clinical and experimental, 64 (4), 216-235. https://doi.org/10.1016/S0011-393X(03)00059-6.

[6] Gilbert, P. E., Brushfield, A. M. 2009. The role of the CA3 hippocampal subregion in spatial memory: A process oriented behavioral assessment. Prog Neuropsychopharmacol Biol Psychiatry. 33, 774-81.

[7] Last RJ. 10th ed. Philadelphia: Churchil-Livingstone; 1999. Hippocampal anantomy; p. 460.

[8] Hippocampus \& Limbic circuit. 39th ed. Elsevier's Sciences; 2003. Grey's Anatomy; pp. 404-10.

[9] Vyas, Y., Montgomery, J. M., \& Cheyne, J. E. (2020). Hippocampal Deficits in Amyloid- $\beta$-Related Rodent Models of Alzheimer's Disease. Frontiers in neuroscience, 14, 266.

[10] X. Wang, Y. Yu, W. Zhao et al., 2018 "Altered whole-brain structural covariance of the hippocampal subfields in subcortical vascular mild cognitive impairment and amnestic mild cognitive impairment patients," Frontiers in Neurology, vol. 9 , p. 342 .

[11] J. Xue, H. Guo, Y. Gao et al., 2019 “Altered directed functional connectivity of the hippocampus in mild cognitive impairment and Alzheimer's disease: a resting-state fMRI study," Frontiers in Aging Neuroscience, vol. 11, p. 326.

[12] Balkissoon, R. 2006. A 26-year-old welder with severe nonreversible obstructive lung disease. COFD. 3, 63-67.

[13] Alvarez-Ayuso, E., A Garcia-Sanchez and X Querol. 2007. Adsorption of $\mathrm{Cr}$ (VI) from synthetic solutions and electroplating wastewaters on amorphous aluminium oxide. $J$. Hazard. 142, 191-198.

[14] He, S., Zhang, A., Niu, Q., Wang, S., and Chen, Y. 2003. Alteration of neurobehavioral and autonomic nervous function in aluminum electrolytic workers. Health Res. 32, 177-179. 
[15] Choueair, N., Laporte, V., Levy, R., Tranchant, C., Gies, J. P., and Poindron, P. 2006. The role of calcium and magnesium ions in uptake of amyloid peptides by microglial cells. Int. $J$. Immunopathol. Pharmacol. 19, 683-696 [16] Jiang, F., Li, W. P., Kwiecien, J., and Tumbull, J. 2006. A study of the purine derivative AIT-082 in G93A SODl transgenic mice. Int. J. Immunopathoi Pharmacol. 19, 489-498.

[16] Jiang, F., Li, W. P., Kwiecien, J., and Tumbull, J. 2006. A study of the purine derivative AIT-082 in G93A SODl transgenic mice. Int. J. Immunopathoi Pharmacol. 19, 489498.

[17] Staibano, S., Mascolo, M., Tranfa, F., Salvatore, G., Mignogna, C. and Bufo, P. 2006. Tumor infiltrating lymphocytes in uveal melanoma: a link with clinical behaviour? Int. J. Immunopathoi Pharmacol. 19, 171-180.

[18] Niu, Q., Niu, P., and He, S. 2004. Effect of Gastrodia elata on learning and memory impairment induced by aluminum in rats. Health Res. 33, 45-48.

[19] Wolozin, B., Wang, S. W., Li, N. 2007. Simvastatin is associated with a reduced incidence of dementia and Parkinson's disease. BMC Med. 5, 20.

[20] Newman, T. and Hulley, S. 1996. Carcinogenicity of lipidlowering drugs. JAMA. 275, 55-60.

[21] Cucchiara, B. and Kasner, S. 2001. Use of statins in CNS disorders. J Neurol Sci. 187, 81-9.

[22] Ostrowski, S. M. 2016. Simvastatin inhibits protein isoprenylation in the brain. Neuroscience 329, 264-274,

[23] Pedrini, S. 2005. Modulation of statin-activated shedding of Alzheimer APP ectodomain by ROCK. PLoS Med. 2, e18.

[24] Su, Bin and Chen, Xiaoyang. (2020) Current Status and Potential of Moringa oleifera Leaf as an Alternative Protein Source for Animal Feeds, Frontiers in Veterinary Science; 7 [53].

[25] Bamishaiye EI, Olayemi FF, Awagu EF, Bamshaiye OM. Proximate and phytochemical composition of Moringa oleifera leaves at three stages of maturation. Adv J Food Sci Tech. (2011) 3: 233-7.

[26] Ozioko OM, Ozioko US, Mba CE, Ozor II and Egwuatu D. Anxiolytic and curative effect of Solanum macrocarpon leaves extract on acetaminophen induced brain injury in adult Wistar rats. J Pharmacogn Phytochem. 2020; 9 (3): 205-212).

[27] Ullah, R.; Khan, M.; Shah, S. A.; Saeed, K.; Kim, M. O. 2019, Natural antioxidant anthocyanins-A hidden therapeutic candidate in metabolic disorders with major focus in neurodegeneration. Nutrients 11, 1195 .

[28] Moneim, A. E. A. Oxidant/antioxidant imbalance and the risk of Alzheimer's disease. Curr. Alzheimer Res. 2015, 12, 335349 .

[29] Wojsiat, J.; Zoltowska, K. M.; Laskowska-Kaszub, K.; Wojda, U. 2018; Oxidant/antioxidant imbalance in Alzheimer's disease: Therapeutic and diagnostic prospects. Oxidative Med. Cell. Longev., 2018, 6435861.
[30] Cicero, A. F.; Fogacci, F.; Banach, 2018 M. Botanicals and phytochemicals active on cognitive decline: The clinical evidence. Pharmacol. Res., 130, 204-212.

[31] Phan, H. T.; Samarat, K.; Takamura, Y.; Azo-Oussou, A. F.; Nakazono, Y.; Vestergaard, M. d. C. 2019, Polyphenols modulate Alzheimer's amyloid beta aggregation in a structuredependent manner. Nutrients11, 756. Craggs, L., and Kalaria. R. N. 2011. "Revisiting dietary antioxidants, neurodegeneration and dementia," NeuroReport, 22 (1), 1-3.

[32] Ganguly, R. and Guha, D. 2008. Alteration of brain monoamines \& EEG wave pattern in rat model of alzheimer's disease \& protection by Moringa oleifera. Indian Journal of Medical Research. 128 (6), 744-751.

[33] Atuadu, Vivian \& Anibeze, Chike \& Elizabeth, Finbarrs-bello \& Esom, Emmanuel \& Okoh, D.. (2019). Protective role of adansonia digitata on lead-induced neurotoxicity in the hippocampus of adult wistar rats. IBRO Reports. 7. 19. 10.1016/j.ibror.2019.09.042.

[34] Ozor II, Ozioko OM, Ozioko US, MbaCE, Abireh IE, Blessed OC. 2020; Microanatomical and Hormonal Studies of the Effects of Aqueous Cannabis sativa Leaf Extract on the Testis of Adult Wistar Rats. SSR Inst. Int. J. Life Sci., 6 (4): 25942600 .

[35] Onyinye Mary Ozioko, Uche Sebastine Ozioko, Christian Ejuiwa Mba, Vivian Atuadu, Ifeanyi Anthony Egwuatu, Anayo Okoro. 2020; Curative Effect of Aqeous Leaf Extract of Solanum Macrocarpon on Acetaminophen Induced Nephrotoxicity on Adult Wistar Rats. World Journal of Pharmaceutical Research 9 (6), 158-172. DOI: 10.20959/wjpr20206-17650.

[36] Ozor II, Agwagu ZN, Finbarrs-Bello E, Ozioko OM, Ozioko US, Mgbachi LC. Morphohistological Effect of Prenatal Alcohol Exposure on the Hippocampus of New Born Wistar Rat. Trop J Nat Prod Res. 2020; 4 (8).

[37] Andrade-Moraes, C. H., Oliveira-Pinto, A. V., Castro-Fonseca, E., da Silva, C. G., Guimarães, D. M., Szczupak, D., ParenteBruno, D. R., Carvalho, L. R., Polichiso, L., Gomes, B. V., Oliveira, L. M., Rodriguez, R. D., Leite, R. E., FerrettiRebustini, R. E., Jacob-Filho, W., Pasqualucci, C. A., Grinberg, L. T., \& Lent, R. (2013). Cell number changes in Alzheimer's disease relate to dementia, not to plaques and tangles. Brain: $a$ journal of neurology, 136 ( $\mathrm{Pt} 12), \quad 3738-3752$. https://doi.org/10.1093/brain/awt273).

[38] Manuela, P., Alin, C., Ioannis, M., Dimitrios, F. \& Stavros, B. 2012. Hippocampal neuronal loss in the cal and ca3 areas of alzheimer's disease patients. Psychiatria Danubina. 24 (2), 152-158.

[39] Kumar, S. E. P., Bairy, K. L., Nayak, V., Reddy, S. K., Kiran, A. and Ballal, A. 2019. Amelioration of Aluminium Chloride induced neurotoxicity by combination of Rivastigmine and Memantine with Artesunate in Albino wistar Rats. Biomed Pharmaco J. 12 (2).

[40] Murphy, M. P., \& LeVine, H., 3rd (2010). Alzheimer's disease and the amyloid-beta peptide. Journal of Alzheimer's disease: $J A D, 19$ (1), 311-323. https://doi.org/10.3233/JAD-2010-1221. 